PROCEEDINGS OF THE AMERICAN MATHEMATICAL SOCIETY

Volume 124, Number 2, February 1996

\title{
INVARIANT POINTS OF MAPS BETWEEN GRASSMANNIANS
}

\author{
KALYAN MUKHERJEA AND PARAMESWARAN SANKARAN
}

(Communicated by Thomas Goodwillie)

\begin{abstract}
We show that for a very large class of integers $l<k<n$ and any map $f: G_{k}\left(\mathbb{R}^{n}\right) \rightarrow G_{l}\left(\mathbb{R}^{n}\right)$ between Grassmannians, there is some $k$-plane of $\mathbb{R}^{n}$ which is mapped into a subspace of itself.
\end{abstract}

\section{INTRODUCTION}

For positive integers $l<k<n$ and a map $f: G_{k}\left(\mathbb{R}^{n}\right) \rightarrow G_{l}\left(\mathbb{R}^{n}\right)$ of Grassmannians, a point $\pi \in G_{k}\left(\mathbb{R}^{n}\right)$ - that is, a $k$-plane in $\mathbb{R}^{n}$ —will be called an invariant point of $f$ if $f(\pi) \subset \pi$. Our interest in such "invariant subspaces" $(f(\pi) \subset \pi !)$ was aroused by questions posed by Mathematical Economists; invariant points appear to play some role in the theory of General Equilibrium.

We will prove the following surprisingly strong result on the existence of invariant points for maps between Grassmannians.

Theorem 1.1. Let $s$ be a positive integer and suppose that $2^{s-1}<n<2^{s}$. If the integers $k, l$ satisfy the inequalities $1 \leq l<k \leq \frac{n}{2}$ and $k+2 l-1 \leq n$ and if $(n, k) \neq\left(2^{s}-1,2\right)$, then any continuous map $f: G_{k}\left(\mathbb{R}^{n}\right) \rightarrow G_{l}\left(\mathbb{R}^{n}\right)$ has an invariant point; i.e. there is some $k$-plane $\pi$ in $\mathbb{R}^{n}$ such that $f(\pi) \subset \pi$.

We would like to thank the referee for some valuable suggestions.

\section{Maps Between Grassmannians}

Throughout $H^{*}$ will denote cohomology with coefficients in $\mathbb{F}_{2}$, the field with two elements. We need a result on the nature of the morphism induced on cohomology by a map between Grassmannians.

Theorem 2.1. Let $f: G_{k}\left(\mathbb{R}^{n}\right) \rightarrow G_{l}\left(\mathbb{R}^{n}\right)$ be any map. Suppose that the integers $n, k, l$ satisfy the following conditions:

- $0<2^{s-1}<n \leq 2^{s}$;

- $1 \leq l<k \leq \frac{n}{2}$

- $k+2 l-1 \leq n$.

Received by the editors April 5, 1994 and, in revised form, September 21, 1994

1991 Mathematics Subject Classification. Primary 55M20, 54H25.

Key words and phrases. Fixed-point theory, Grassmannians, invariant points, mod 2 Steenrod algebra.

(C)1996 American Mathematical Society 
(i) If $n \neq 2^{s}$ and $(n, k) \neq\left(2^{s}-1,2\right)$, then $f^{*}: \widetilde{H}^{*}\left(G_{l}\left(\mathbb{R}^{n}\right)\right) \rightarrow \widetilde{H}^{*}\left(G_{k}\left(\mathbb{R}^{n}\right)\right)$ is the zero homomorphism on reduced cohomology.

(ii) If $n=2^{s}$ or $(n, k)=\left(2^{s}-1,2\right)$, then either $f^{*}$ is zero or $f^{*}\left(w_{i}\left(\gamma_{n, l}\right)\right)=$ $\left(\begin{array}{l}l \\ i\end{array}\right)\left(w_{1}\left(\gamma_{n, k}\right)\right)^{i}$ where $\gamma_{n, p}$ is the canonical p-plane bundle on $G_{p}\left(\mathbb{R}^{n}\right)$ and $w_{i}$ is the ith Stiefel-Whitney class.

This is proved in an entirely elementary manner in $[\mathrm{K}-\mathrm{S}]$ but since this source may be inaccessible to many we are sketching a proof using a more easily available paper $[\mathrm{P}]$ of Patterson, although the techniques used by Patterson are rather more sophisticated. The basic idea is that $f^{*}$ is not just a map of cohomology rings but is an $\mathcal{A}(2)$-module homomorphism where $\mathcal{A}(2)$ is the mod 2 Steenrod algebra. We will be looking at homomorphisms between cohomology algebras of spaces (whether or not they are induced by a map) and say that such a homomorphism is an $\mathcal{A}(2)$-map if it is a homomorphism of the cohomology rings and an $\mathcal{A}(2)$-module homomorphism.

Using Patterson's work one can classify the $\mathcal{A}(2)$-maps between the cohomologies of the infinite Grassmannians $G_{k}\left(\mathbb{R}^{\infty}\right)=B O(k)$ and $B O(l)$. We explain this briefly. The cohomology of $B O=\bigcup_{p} B O(p)$ is a polynomial algebra with the universal Stiefel-Whitney classes $\left\{w_{i}\right\}_{i \geq 1}$ as generators. The inclusion map $B O(p) \rightarrow B O$ pulls back $w_{i}$ to the $i$ th Stiefel-Whitney class of the canonical $p$-plane bundle $\gamma_{\infty, p}$ on $G_{p}\left(\mathbb{R}^{\infty}\right)$. We will denote the images of $w_{i}$ in $B O(l)$ (resp. $B O(k)$ ) by $x_{i}$ (resp. $y_{i}$ ) and with this notation $H^{*}(B O(l))$ (resp. $\left.H^{*}(B O(k))\right)$ is generated as an algebra by $x_{1}, \ldots, x_{l}$ (resp. $\left.y_{1}, \ldots, y_{k}\right)$. Note that $x_{i}$ (resp. $\left.y_{i}\right)$ is zero if $i>l$ (resp. $i>k$ ).

Let $\mathbb{P} \cong B O(1)$ be the infinite-dimensional projective space; its cohomology is a polynomial algebra on a single element $t$ of degree 1 .

Patterson shows that if

$$
\alpha: H^{*}(B O) \rightarrow H^{*}(\mathbb{P} \stackrel{k \text { factors }}{\times \cdots \times} \mathbb{P})=\mathbb{F}_{2}\left[\left[t_{1}, \ldots, t_{k}\right]\right]
$$

is an $\mathcal{A}(2)$-map, then

$$
\alpha\left(1+w_{1}+w_{2}+\cdots\right) \mapsto \prod_{i_{1}<\cdots<i_{r}}\left(1+t_{i_{1}}+\cdots+t_{i_{r}}\right)^{n\left(i_{1}, \ldots, i_{r}\right)}
$$

where $t_{j}$ is the generator in the $j$ th factor, $1 \leq i_{1}<\cdots<i_{r} \leq k, 1 \leq r \leq k$, and the $n\left(i_{1}, \ldots, i_{r}\right)$ are 2 -adic integers.

From this one easily checks that $\alpha$ will kill $w_{i}$ for all $i>l$ if and only if $n\left(i_{1}, \ldots, i_{r}\right)$ 's are nonnegative integers whose sum is less than or equal to $l$. This gives us all possible $\mathcal{A}(2)$-homomorphisms

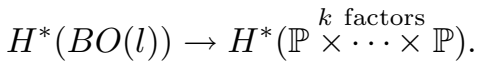

Since $H^{*}(B O(k))$ is the set of elements on the right-hand side which remain fixed under the action of the permutation group $\mathfrak{S}_{k}$ acting on the factors, we can deduce the following result (which is Theorem 2 of $[\mathrm{K}-\mathrm{S}]$ ).

Proposition 2.2. Suppose $2^{s-1}<n \leq 2^{s}$ and suppose that $k, l$ satisfy the inequalities $1 \leq l<k \leq \frac{n}{2}$. If $\phi: H^{*}(B O(l)) \rightarrow H^{*}(B O(k))$ is an $\mathcal{A}(2)$-map, then either $\phi$ is zero in positive dimensions or for some integer $1 \leq t \leq l, \phi\left(x_{i}\right)=\left(\left(\begin{array}{l}t \\ i\end{array}\right)\right) y_{1}^{i}$.

In particular, if $f: B O(k) \rightarrow B O(l)$ is a map, then either $f^{*}$ is trivial on reduced cohomology or $f^{*}\left(w_{i}\left(\gamma_{\infty, l}\right)\right)=\left(\left(\begin{array}{c}t \\ i\end{array}\right)\right)\left(w_{1}\left(\gamma_{\infty, l}\right)\right)^{i}$ for some integer $1 \leq t \leq l$. 
We use this result to deduce Theorem 2.1. Let $f: G_{k}\left(\mathbb{R}^{n}\right) \rightarrow G_{l}\left(\mathbb{R}^{n}\right)$ be any map. Let $j_{p}: G_{p}\left(\mathbb{R}^{n}\right) \rightarrow B O(p)$ be the inclusion map. It is known that $j_{p}^{*}$ is an isomorphism on cohomology for dimensions up to $n-p$. Since $l \leq 2 l-1 \leq$ $n-k$, we can define a map $\phi: H^{*}(B O(l)) \rightarrow H^{*}(B O(k))$ by setting $\phi\left(x_{i}\right)=$ $\left(j_{k}^{*}\right)^{-1} f^{*} j_{l}^{*}\left(w_{i}\left(\gamma_{\infty, l}\right)\right)$.

A brief calculation shows that the homomorphism $\phi$ so defined is an $\mathcal{A}(2)$-map and hence by Proposition 2.2 either $f^{*}=0$ in positive dimensions or $f^{*}\left(w_{i}\left(\gamma_{n, l}\right)\right)=$ $\left(\left(\begin{array}{l}t \\ i\end{array}\right)\right)\left(w_{1}\left(\gamma_{n, k}\right)\right)^{t}$ for some $t \leq l$.

Recall that the height of a cohomology class $x$ is the largest integer $h$ such that $x^{h} \neq 0$. For $2^{s-1}<n \leq 2^{s}$ Stong ([St]) has shown that the height of $w_{1}\left(\gamma_{n, k}\right)$ is $2^{s}-2$ if $k=2$ or if $(n, k)=\left(2^{s-1}+1,3\right)$ and that otherwise it is equal to $2^{s}-1$. We are now ready to prove Theorem 2.1.

Assume that $f^{*} \neq 0$. Then writing $w$ for the total Stiefel-Whitney class, our previous arguments show that $f^{*} w\left(\gamma_{n, l}\right)=\left(1+w_{1}\left(\gamma_{n, k}\right)\right)^{t}$ for some $1 \leq t \leq l$. We will write $z$ for $w_{1}\left(\gamma_{n, k}\right)$, for the sake of brevity.

The fact that $\gamma_{n, l}$ and $\gamma_{n, l}^{\perp}$ are stably complementary bundles and the rank of the latter is $(n-l)$ yields

$$
f^{*}\left(1+w\left(\gamma_{n, l}^{\perp}\right)\right)=(1+z)^{-t}=1+a_{1} z+\cdots+a_{p} z^{p}
$$

where the $a_{i}$ 's are in $\mathbb{F}_{2}, a_{p} \neq 0$ and $p \leq(n-l)$. Since $w\left(\gamma_{n, l}\right) \cdot w\left(\gamma_{n, k}^{\perp}\right)=1$, this gives

$$
\left(1+\left(\begin{array}{l}
t \\
1
\end{array}\right) z+\cdots+\left(\begin{array}{l}
t \\
t
\end{array}\right) z^{t}\right) \cdot\left(1+a_{1} z+\cdots+a_{p} z^{p}\right)=1 .
$$

In particular, since $a_{p} \neq 0$ we conclude that $z^{p+t}=0$. Since $p+t \leq(n-l)+l=n$, this contradicts Stong's result unless $n=2^{s}$ or $(n, k)=\left(2^{s}-1,2\right)$. When $k=2$ both $l$ and $t$ are bounded by 1 . So either $f^{*}=0$ or $t=l=1$. Finally if $n=2^{s}$ and $k \geq 3$ Stong's result implies $z^{n-1} \neq 0$ and again this is contradicted by $z^{p+t}=0$ unless $t=l$. This completes the proof.

\section{Proof of Theorem 1.1}

We proceed along the lines of Lefschetz's proof of his fixed point theorem. For brevity we will write $K=G_{k}\left(\mathbb{R}^{n}\right)$ and $L=G_{l}\left(\mathbb{R}^{n}\right)$. Let $V=\{(x, y) \in K \times L \mid y \subset$ $x\}$. Then for any map $f: K \rightarrow L$ the set of invariant points of $f, \Sigma_{f}=\{x \in$ $K \mid f(x) \subset x\}$, is the preimage of $V$ by the map $1_{K} \times f$.

It is easy to identify $V$ with a flag manifold. If $(x, y) \in V$, let us denote the orthogonal complement of $y$ in $x$ by $x \ominus y$. Then $(x, y) \mapsto\left(y, x \ominus y, x^{\perp}\right)$ gives a diffeomorphism of $V$ onto the flag manifold $\mathcal{F}(l, k-l, n-k)$ which has dimension $s=l(n-l)+(k-l)(n-k)$. Let $v \in H^{m-s}(K \times L)$ be the Poincaré dual of the $\mathbb{Z}_{2}$-fundamental class of $V$ where $m=k(n-k)+l(n-l)$ is the dimension of $K \times L$. Then it is well known that

$$
v \in \operatorname{Image}\left\{H^{*}(K \times L, K \times L \backslash V) \rightarrow H^{*}(K \times L)\right\} .
$$

Hence if $f$ has no invariant points, then $\left(1_{K} \times f\right)^{*}(v)=0$. Now for the dimension restrictions provided by our hypotheses, Theorem 2.1 affirms that the cohomology homomorphism induced by $f$ is zero and hence independent of the map $f$. Therefore $\left(1_{K} \times f\right)^{*}(v)=\left(1_{K} \times \star\right)^{*}(v)$ where $\star: K \rightarrow L$ is the map which takes each $k$-plane to a fixed $l$-plane, say the one spanned by the first $l$ elements of the standard basis of $\mathbb{R}^{n}$. But $\Sigma_{\star}$ is obviously a Schubert cell and since this is one of the generators 
of the homology of $K$, its Poincaré dual $\xi$ is nonzero. So $\left(1_{K} \times f^{*}\right)(v)=\xi \neq 0$ and hence $f$ has an invariant point.

\section{Concluding Remarks}

We begin by discussing the sharpness of our result; we are well aware that sharper bounds may be possible.

4.1. If $k>\frac{n}{2}$, then taking any $k$-plane to its orthogonal complement gives a map without invariant points.

4.2. The exclusion $\left(2^{s}-1,2\right) \neq(n, k)$ is necessary. Let $\mathcal{C}$ be the 8 -dimensional Cayley division algebra and consider $\mathbb{R}^{7}$ as the subspace of purely imaginary Cayley numbers. Then, if $\pi$ is a 2-plane in $\mathbb{R}^{7}$, choose an orthonormal basis $\{x, y\}$. Define $f: G_{2}\left(\mathbb{R}^{7}\right) \rightarrow G_{1}\left(\mathbb{R}^{7}\right)=\mathbb{R P}_{6}$ by defining $f(\pi)$ to be the space spanned by the nonzero element $x \cdot y$. This is well defined and since $x \cdot y$ is orthonormal to both $x$ and $y, f$ has no invariant point.

4.3. Using the 3 -fold vector product on $\mathbb{R}^{8}$ defined in $[\mathrm{Z}]$ one can produce maps $G_{3}\left(\mathbb{R}^{8}\right) \rightarrow G_{1}\left(\mathbb{R}^{8}\right)=\mathbb{R P}_{7}$ without any invariant points; so the condition $n \neq 2^{m}$ is also necessary.

4.4. One might ask also if there are conditions which will ensure that all maps $g: G_{l}\left(\mathbb{R}^{n}\right) \rightarrow G_{k}\left(\mathbb{R}^{n}\right)$ (notice that we still have $l<k$ ) will admit a point $\pi$ such that $\pi \subset f(\pi)$. (These are fixed-points in the sense of multi-valued maps.) For $l=1$ there is generally no such fixed-point property; whenever $k$ is less than the Radon-Hurwitz number, $\rho(n)$, such maps can be easily constructed.

Finally we would like to point out that Theorem 1.1 does not generalize to Grassmannians of Banach spaces.

Proposition 4.5. Let $\ell_{2}$ be a separable Hilbert space of infinite-dimension (over $\mathbb{R}$ or $\mathbb{C})$. Then for any two integers $k$ and $l$ there is a map $f: G_{k}\left(\ell_{2}\right) \rightarrow G_{l}\left(\ell_{2}\right)$ such that for any $k$-plane, $\pi$ and $f(\pi)$ are linearly disjoint.

Proof. Consider the bundle $\gamma_{k}^{\perp} \rightarrow G_{k}\left(\ell_{2}\right)$ whose fibre over a $k$-plane $\pi$ is the set of vectors orthogonal to $\pi$. This is a vector bundle with an infinite-dimensional Hilbert space as fibre. As a consequence of Kuiper's theorem [Ku] affirming the contractibility of the general linear groups of infinite-dimensional Hilbert spaces, the bundle $\gamma_{k}^{\perp}$ is isomorphic to a product vector bundle. Choose sections $s_{1}, \ldots, s_{l}$ of $\gamma_{k}^{\perp}$ which are linearly independent at every point and define $f: G_{k}\left(\ell_{2}\right) \rightarrow G_{l}\left(\ell_{2}\right)$ by setting $f(\pi)$ to be the linear span of $s_{1}(\pi), \ldots, s_{l}(\pi)$. Clearly, $f(\pi) \cap \pi=\{0\}$.

This result is probably false if we replace $\ell_{2}$ by some other Banach space of infinite dimension. There are Banach spaces (for instance, $c_{0} \oplus l_{2}$ ) whose general linear groups have nontrivial homotopy; so Kuiper's theorem cannot be invoked. More importantly, it is not clear how to define a bundle of "complementary subspaces" to take the place of $\gamma_{k}^{\perp}$ when dealing with general Banach spaces. On the other hand we also do not know if Theorem 1.1 (or some variant thereof) holds for any Banach space. 


\section{REFERENCES}

[K-S] J. Korbas and P. Sankaran, On continuous maps between Grassmann manifolds, Proc. Indian Acad. Sci. Math. Sci. 101 (1991), 111-120. MR 92f:55026

$[\mathrm{Ku}] \mathrm{N}$. H. Kuiper, The homotopy type of the unitary group of infinite dimensional Hilbert space, Topology 3 (1965), 19-30. MR 31:4034

[P] R. R. Patterson, The square preserving algebra endomorphisms of $H^{*}\left(B O ; Z_{2}\right)$ Quart. J. Math. Oxford Ser. (2) 29 (1978), 225-240. MR 80g:55033

[St] R. E. Stong, Cup products in Grassmannians Topology Appl. 13 (1982), 103-113. MR 83c: 55003

[Z] P. Zvengrowski, A 3-fold vector product in $\mathbb{R}^{8}$, Comment. Math. Helv. 40 (1966), 149-152. MR 32:8343

Statistics-Mathematics Division, Indian Statistical Institute, 203 Barrackpore Trunk Road, Calcutta 700035, India

E-mail address: kalyan@isical.ernet.in

SPIC Science Foundation, 92 G. N. Chetty Road, T. Nagar, Madras 600017, India

E-mail address: sankaran@ssf.ernet.in 